\title{
Estudio de sensibilidad antibiótica en las bacteriemias de un hospital secundario
}

\author{
R. PAZOS AÑÓN, R. FERNÁNDEZ RODRIGUEZ, A. TINAJAS', C. NANÍN, \\ M. BUSTILLO, I. PAZ ${ }^{1}$, R. BARREIRO, P. GAYOSO DIZ ${ }^{2}$

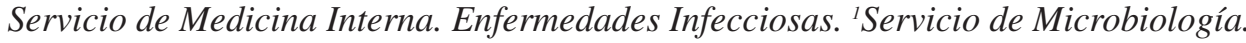 \\ ${ }^{2}$ Unidad de Investigación. Cristal-Piñor Hospitais. Ourense
}

ANTIMICROBIAL SUSCEPTIBILITY OF THE BLOODSTREAM
INFECTIONS: A STUDY IN A NONTEACHING HOSPITAL

\section{RESUMEN}

Objetivo: Conocer los patógenos prevalentes y los patrones de resistencia de las bacteriemias nosocomiales y adquiridas.

Material y métodos: Estudio prospectivo de 310 bacteriemias "significativas" detectadas en un hospital no universitario en el periodo octubre 2000-2001. Los hemocultivos se detectaron por el sistema Bact-Alert y la identificación definitiva se efectuó con el sistema MicroScan, con estudio de sensibilidad por el método de microdilución de referencia (NCLSS). Se estudiaron combinaciones "centinela" con relevancia clínica. El análisis estadístico utilizó el programa SPSS. El análisis univariante utilizó para variables cuantitativas t Student y para cualitativa test X2 o t Fisher.

Resultados: Los gram positivos y las levaduras predominaron en las nosocomiales. Hubo un $24 \%$ de MARSA y un $14 \%$ de neumococos penicilina resistentes. La vancomicina mantuvo una sensibilidad universal. Los gram negativos dominaron en las comunitarias, E. coli $(40 \%)$ con una prevalencia de EBSL $<2 \%$. Destacamos las tasas de multiresistencia para $P$. aeruginosa en las unidades de agudos. Un análisis de regresión logística reveló al tratamiento empírico inadecuado como un factor independiente de mortalidad (OR 2,6).

Conclusiones: La necesidad de un sistema de vigilancia que permita conocer la prevalencia y resistencia de los microorganimos locales y establecer terapéuticas empíricas adecuadas que permitan reducir la mortalidad.

PALABRAS CLAVE: Bacteriemia. Sensibilidad antibiótica. Mortalidad.

\begin{abstract}
Background: To try established antimicrobial susceptibility patterns and frequency of both nosocomial and community-acquired bloodstream infections and and to try identified the pronostic factors that can be modified.

Material and methods: A prospective study of 310 bloodstream infections with clinical significance detected in a non teaching hospital over period from October 2000-2001. A blood culture were idenfied by BactAlert system and the confirmation was performed by MicroScan system; an antimicrobial susceptibility test was performed by reference microdilution methods as described by NCCLS. We studied sentinel antimicrobial/organism combinations with potencial clinical importance.

Data were computerized using SPSS. Qualitative variables were compared using the X2 test or the Fisher exact test, and quantitative variables with $t$ Student or ANOVA.

Results: Gram positive and Candida were frequently recovered in nosocomial bloodstreams. The proportion of oxacillin-resistant $\mathrm{S}$. aureus isolates was $24 \%$ and the penicillin resistant pneumococci was $14 \%$. Vancomycin was universal active against gram positive. Gram negatives were often recovered in community bloodstream. The proportion of EBSL E. coli isolates was $<2 \%$ and the proportion of multiresistance Pseudomonas aeruginosa was higher among UCI isolates.

An independent risk factors for death identified after multivariate análisis was the inapropiate antimicrobian therapy OR 2.6.

Conclusions: Ongoing surveillance of microbial pathogens and their resistance profiles is essential on local escale and permit the selection of appropiate antibiotic therapy which would be reduce the mortality.
\end{abstract}

KEY WORDS: Bloodstream. Antimicrobial susceptibility. Mortality.

Pazos Añón R, Fernández Rodriguez, R, Tinajas A, Consuelo Nanín, Bustillo M, Paz I, Barreiro R, Gayoso Diz P. Estudio de sensibilidad antibiótica en las bacteriemias de un hospital secundario. An Med Interna (Madrid) 2004; 21: 483-487.

\section{INTRODUCCIÓN}

Se han publicado estudios que muestran la presencia de cambios continuos en los microorganismos aislados en los hemocultivos, lo que obliga a establecer sistemas de vigilancia continua (1). La mayoría de los estudios microbiológicos han sido desarrollados en hospitales universitarios y tenemos la impresión que la extrapolación de dichos resultados a hospitales más pequeños podría estar exagerando los rangos de resistencia y conducir a un abuso de los antibióticos de amplio espectro (2).

Trabajo aceptado: 5 de mayo de 2004 
El aumento de las resistencias antibióticas, condicionado por el uso inadecuado de antibióticos, es un hecho conocido que obliga a utilizar antibióticos más caros, aumenta las estancias hospitalarias, la mortalidad y en definitiva, los costes en salud $(3,4)$. Se impone por tanto, un uso racional de los mismos por los profesionales sanitarios (5).

El objetivo de este estudio es conocer los microorganismos más prevalentes y las resistencias antibióticas clínicamente significativas en nuestro medio categorizadas según su origen de adquisición.

\section{MATERIAL Y MÉTODOS}

El Hospital Cristal Piñor es centro de referencia para la provincia de Ourense, dispone de 650 camas y carece de los servicios de Cirugía Cardio-torácica y de programa de transplante (a excepción de médula ósea autólogo). Proponemos un estudio prospectivo de las bacteriemias clínicamente significativas detectadas en el periodo de octubre 2000- octubre 2001. La detección de los casos se iniciaba cuando el laboratorio de microbiología comunicaba la existencia de un hemocultivo positivo y se catalogaba como clínicamente significativa después de la evaluación conjunta del microbiólogo y del clínico responsable de la recogida de datos.

Se utilizaron definiciones de consenso recogidas en la literatura y en un estudio previo de los autores 6 Se consideró bacteriemia al aislamiento de uno o varios microorganismos en hemocultivos, bien por primera vez o repetidamente dentro de los siete dias siguientes a uno primero positivo, siempre que se tratase del mismo microorganismo y en el contexto clínico del paciente. Se consideró adquisición nosocomial, si se detectaba en las 72 horas posteriores al ingreso hospitalario. Se consideró comunitaria cuando se detectaba en las primeras 72 horas del ingreso o 5 dias despues del alta.

Cuando el paciente fallecía dentro de los 7 días posteriores al desarrollo de la septicemia por causas relacionadas con la misma, se consideró muerte directamente relacionada con la septicemia; en aquellos casos en los que la causa del fallecimiento no era evidente se consideró a la septicemia como la causa de la misma. Cuando los datos clínicos y/o microbiológicos no justificaron la causa de la muerte, se consideró que ésta no estaba directamente relacionada. El tratamiento antibiótico empírico, previo a conocer el germen y su sensibilidad, se consideró apropiado cuando fue utilizado algún antibiótico al que el germen se mostró sensible, en las dosis y vía adecuadas.

Fueron analizados 310 hemocultivos detectados por el sistema BACTEC-Alert y efectuada la identificación definitiva con antibiograma por el sistema MicroScan (Dade Behring). Los test de sensibilidad se realizaron por métodos de microdilución de referencia como se estblece en NCSS (7) Se testaron los siguientes antibióticos según el aislamiento fuese un gram positivo o un gram negativo: penicilina, ampicilina, oxacilina, piperacilina-tazobactam, amoxi-clavulánico, cefuroxima, cefotaxima, ceftazidima, imipenem, tobramicina, gentamicina, ciprofloxacina, eritromicina, clindamicina, rifampicina, TMPSMZ (trimetoprim-sulfametoxazol) y vancomicina. Se estudiaron combinaciones antibiótico-resistencia "centinela" en base a su importancia clínica : Staphylococcus aureus y Staphylococcus coagulasa negativos (SCN), Neumococo resistente a penicilina, Enterobacterias resistentes a fluoroquinolonas o cefalosporinas, Gram negativos resistentes a aminoglucósidos, Pseudomonas resistente a ceftazidima, E coli y Kklebsiella pneumoniae resistentes a cefalosporina y, Enterococo resistente a vancomicina.

\section{ANÁLISIS ESTADÍSTICO}

Para el análisis de los datos se utilizó el programa SPSS/PC. Se efectuó el estudio descriptivo de las variables estudiadas, expresando el resultado como $n^{\circ}$ y \%. Para el análisis de las variables cuantitativas se utilizó la media con un IC del 95\%. El análisis bivariante utilizó para variables continuas, los valores medios calculados con el test t Student para muestras independientes después de la corrección por igualdad de varianzas. Las diferencias en las proporciones se compararon con test $\mathrm{X}^{2}$ o test de Fisher (para frecuencias $<5$ ), considerando asociaciones estadísticamente significativas aquellas con un nivel de significación $\mathrm{p}<0,05$.

Se efectuó un análisis univariante para determinar los factores asociados con mortalidad por bacteriemia, la fuerza de asociación entre una única variable pronóstica se expresó como OR e IC $95 \%$.

\section{RESULTADOS}

De los 310 aislamientos, un $53 \%$ fueron gram positivos y un $45,5 \%$ gram negativos. Los patógenos más prevalentes fueron $E$. coli, SCN, S. aureus y enterobacterias; representando los 3 primeros el $63 \%$ del total. A distancia las bacteriemias polimicrobianas $(9,4 \%)$, anaerobios $(4,2 \%)$ y levaduras $(3,2 \%)$.

\section{COMPARATIVA DE MICROORGANISMOS NOSOCOMIALES Y COMUNITARIOS}

Un 54\% de los aislamientos fueron de origen nosocomial y un $46 \%$ comunitario, según distribución adjunta (Tabla I y Fig. 1). Los gram positivos y las levaduras tuvieron un origen predominantemente nosocomial. El S. aureus tuvo una distribución similar en cuanto al origen, mientras SCN fue de predominio nosocomial.

Los gram negativos dominan en las comunitarias, con $E$. coli a la cabeza, y a excepción de $P$. aeruginosa de origen nosocomial. La prevalencia de $\mathrm{S}$ aureus oxacilin-resistentes es muy similar entre los aislamientos comunitarios y nosocomiales. Los neumococos resistentes a la penicilina fueron todos comunitarios.

En general, aunque los datos son muy limitados por el tamaño de la muestra, el porcentaje de resistentes fue mayor en los nosocomiales, con la excepción del $E$. coli resistente al ciprofloxacino. A destacar la elevada tasa de resistencias de los aislamientos de Pseudomonas incluso de las de origen comunitario (Fig. 2).

Dentro de los aislamientos nosocomiales se estudiaron 42 episodios desarrollados en la Unidad de Agudos (UCI-Reanimación) intentando explorar la existencia de diferencias sustanciales: 18 aislamientos fueron gram positivos y 24 gram negativos siendo los aislamientos SCN meticilin-resistentes menos frecuentes, pero las cepas de $P$. aeruginosa multiresis- 
TABLA I

COMPARATIVA DE AISLAMIENTOS SEGUN EL ORIGEN

\begin{tabular}{|c|c|c|c|}
\hline & Noscomial & $x^{2}$ & Comunitaria \\
\hline GRAM + & $64,8 \%$ & P 0,000 & $44 \%$ \\
\hline GRAM - & $30,3 \%$ & P 0,000 & 58,0 \\
\hline Anaerobios & $2,8 \%$ & P ns & $0,4 \%$ \\
\hline Levaduras & $10 \%$ & & -- \\
\hline Polimicrobiana & $11,3 \%$ & P ns & $7,7 \%$ \\
\hline E. coli & $14,1 \%$ & $p, 000$ & $40 \%$ \\
\hline Pseudomona & $4,2 \%$ & $\mathrm{fns}$ & $1,8 \%$ \\
\hline Klebsiella & $4,2 \%$ & f ns & $3,6 \%$ \\
\hline Proteus & -- & f ns & $1,4 \%$ \\
\hline Serratia & $0,7 \%$ & $f, 065$ & $3 \%$ \\
\hline S. aureus & $14,1 \%$ & $\mathrm{p}$ ns & $12,5 \%$ \\
\hline $\mathrm{SCN}$ & $38,7 \%$ & $p, 000$ & $10,1 \%$ \\
\hline Pneumococo & $0,7 \%$ & $\mathrm{fns}$ & $3,6 \%$ \\
\hline Enterococo & $3,5 \%$ & f ns & $4,8 \%$ \\
\hline S. viridans & $2,1 \%$ & $\mathrm{fns}$ & $5,4 \%$ \\
\hline
\end{tabular}

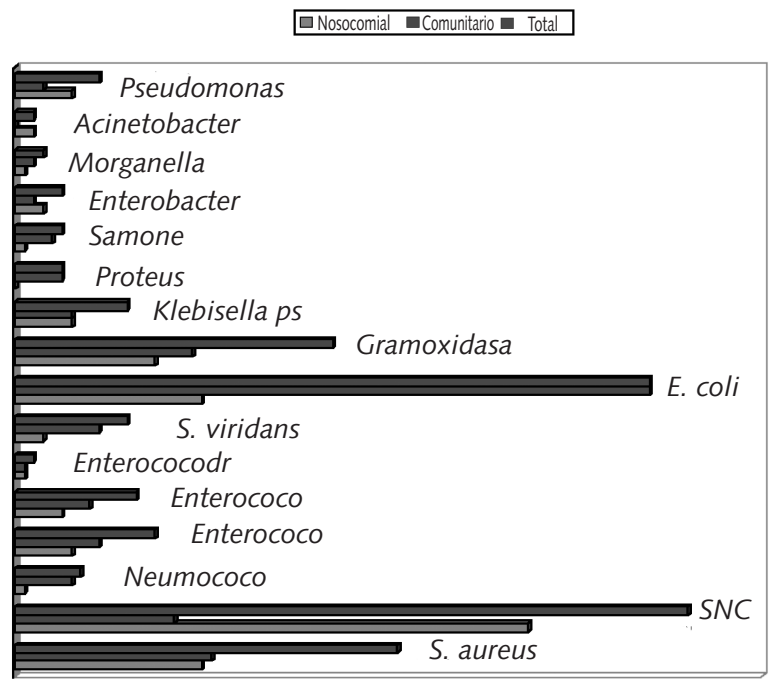

Fig. 1. Distribución de microorganismos.

tentes fueron más prevalentes, al igual que los gram negativos resistentes a cefalosporinas (Tabla II).

El estudio de resistencias reveló:

- Gram positivos:

- Staphylococcus aureus. 41 aislamientos de los cuales $24,4 \%$ fueron resistentes a oxacilina y a betalactámicos. La vancomicina fue activa frente a todos los aislamientos.

- SCN. 71 aislamientos de los cuales un $76 \%$ fueron resistentes a la oxacilina, $98 \%$ a la penicilina, y un $76 \%$ a la cefotaxima y eritromicina respectivamente.

Destacar que un $93 \%$ de los S. aureus y un $67 \%$ de los SCN permanecen sensibles a la gentamicina, aspecto relevante dado su efecto sinérgico con los derivados penicilínicos.

- Enterococcus sp. 15 aislamientos, de ellos un 66,7\% presentaron resistencia a aminoglucósidos, un $66,7 \%$ a la cefota-

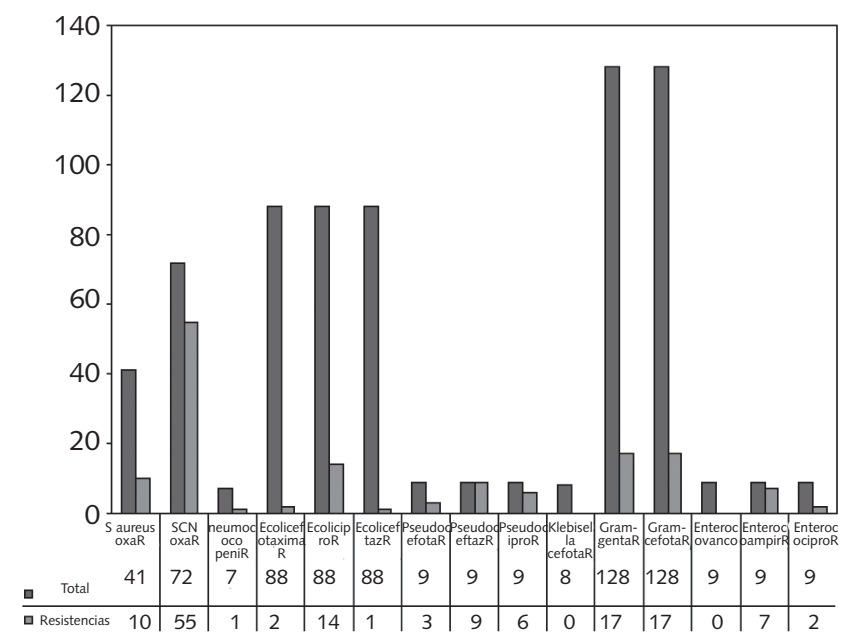

Fig. 2. Resistencia centinela.

xima, un $50 \%$ al ciprofloxacino y un $80 \%$ a la eritromicina. La ampicilina fue sensible en el $87 \%$ de los aislamientos y no se detectó ningún aislamiento resistente a vancomicina.

- Streptococcus pneumoniae. 8 aislamientos de los 14,3\% fueron cepas resistentes a la penicilina, $28,6 \%$ a la eritromicina y un $40 \%$ a la gentamicina. Todos fueron sensibles a la cefotaxima, ciprofloxacino y vancomicina.

- S. viridans. 12 aislamientos con un $91 \%$ de cepas sensibles a penicilina, un $41 \%$ de cepas resistentes a eritromicina y un $20 \%$ a la clindamicina.

- Gram negativos.

- E. coli. 83 aislamientos. Todos fueron sensibles a carbapenems y presentaron elevada sensibilidad a los aminoglucósidos (91\% para la gentamicina y un $100 \%$ a la amikacina). La piperacilina-tazobactam fue la más activa de las penicilinas 
TABLA \|

ESTUDIO DE SENSIBILIDAD EN LA UNIDAD DE AGUDOS

\begin{tabular}{lcl}
\hline & Unidad Agudos-----resto & \\
\hline S. aureus oxaresistente & $2,4 \%$ frente $3,4 \%$ & \\
SCN oxaresistente & $15,7 \%$ frente $31 \%$ & \\
Pneumococo penicilina resistente & ------------------ & \\
E. coli cefotaxima resistente & $0 \%$ frente $0,8 \%$ & \\
E. coli ciprofloxacino resistente & $0 \%$ frente $5,3 \%$ & \\
E. coli ceftazidima resistente & $0 \%$ frente $0,4 \%$ & \\
Pseudomona cefotaxima resistente & $9,5 \%$ frente $1,9 \%$ & p,022 \\
Pseudomona ceftazidima resistente & $7,1 \%$ frente $0 \%$ & \\
Pseudomona ciprofloxacino resistente & $9,5 \%$ frente $1,9 \%$ & \\
Klebsiella cefotaxima resistente & ------------- & \\
Gramnegativos gentamicina resistente & $9,5 \%$ frente $5 \%$ & \\
Gramnegativos cefotaxima resistente & $14,3 \%$ frente $4,2 \%$ & \\
Enterococo ampicilina resistente & $0 \%$ frente $0,7 \%$ & \\
Enterococo vancomicina resistente & & \\
Enterococo ciprofloxacino resistente & $4,8 \%$ frente $1,9 \%$ & \\
\hline
\end{tabular}

(94\% de sensibilidad) y la ceftazidima de las cefalosporinas (no fue testado cefepime). Las cepas mantienen sensibilidad del $84 \%$ al ciprofloxacino y de un $77 \%$ al trimetoprim-sulfametoxazol (TMP-SMZ). Las resistencias para cefotaxima fue del 2,3\% y del $8,1 \%$ para cefuroxima. Estos datos sugieren una baja prevalencia de betalactamasas de espectro ampliado (EBSL).

- Klebsiella sp. 11 aislamientos, con cepas altamente sensibles a derivados penicilínicos (91\% piperacilina-tazobactam) y $100 \%$ a cefotaxima y carbapenem. Se documentó un $8,3 \%$ de resistencias a ciprofloxacino.

- Pseudomonas aeruginosa. 9 aislamientos con $100 \%$ de cepas resistentes a cefotaxima, cefuroxima y un $55 \%$ a ceftazidima. Un 33\% de resistencia a piperacilina-tazobactam, $66,7 \%$ a gentamicina y ciprofloxacino respectivamente. Se mantuvo una sensibilidad del $78 \%$ al imipemen y amikacina.

Los carbapenemes fueron los antibióticos más activos frente a los gram negativos, con la excepción de un $22 \%$ de resistencias para Pseudomonas aeruginosa. Las fluoroquinolonas mostraron una sensibilidad elevada a excepción de la Pseudomonas donde fue sólo del $43 \%$ de los aislamientos. De los aminoglucósidos, la amikacina fue la más activa. Usando la resistencia a ceftazidima y ceftriaxona como marcadores de la presencia de cepas EBSL, para E. coli sólo un 1,2\% de las cepas serian portadoras potenciales de EBSL.

Publicamos una mortalidad relacionada global del 13\% y una cruda del 23\%, similar a otros estudios europeos (16), pero no se identificó ningún germen presentase diferencias estadísticamente significativas en cuanto a la mortalidad que provocaba; sólo el E. coli presentaba las tasas de mortalidad más bajas $(6 \%$ ) con OR 0,3 (IC 95\% 0,12-0,84) La mortalidad se incrementaba ante la presencia de gérmenes resistentes alcanzando un $25 \%$ para S. aureus meti-resistente y un $40 \%$ para Pseudomonas ciproresistentes.

\section{DISCUSIÓN}

La existencia de un sistema de vigilancia de resistencias es fundamental para un manejo adecuado de las infecciones, permitiendo conocer los rangos de infección, los cambios en los patógenos y las tendencias de las resistencias en las infecciones nosocomiales y comunitarias y en base a los mismos diseñar tratamientos empíricos y definir las medidas de control necesarias (9).

Los estudios existentes en la literatura sugieren que los rangos de resistencia antibiótica son mayores en el hospital que en la comunidad y dentro del hospital en las unidades de agudos como consecuencia del efecto de selección que ejerce un gran número de antibióticos administrados a un único paciente (10). Pero, esto ya no es la norma y en nuestro caso se documentan cepas resistentes para oxacilina en el S. aureus comunitaro, o para ciprofloxacino en los aislamientos de $E$. coli y Pseudomonas comunitarios.

Destacar que un $24 \%$ de los aislamientos de los S. aureus fueron meticilin-resistentes, similar a la documentada en otros centros de mayor tamaño $(2,11)$ y con análoga distribución según el origen, probablemente por tratarse de pacientes clasificados como comunitarios pero con múltiples ingresos previos, dato ya documentado en la literatura (12). Existe controversia en el tratamiento, en cuanto a la elección de un betalactámico y/o glicopéptico, en nuestra serie la segunda opción parece más segura ante las elevadas tasas de resistencia a penicilina y betalactámicos. El neumococo sigue siendo una causa de morbimortalidad comunitaria en jóvenes y ancianos, con un aumento en el número de cepas resistentes ( $14 \%$ penicilina), que podría ser reflejo del aumento del espectro antibiótico en pacientes externos (13). Los enterococos resistentes a la vancomicina están en aumento en hospitales grandes y universitarios en donde se han publicado brotes 
de difícil tratamiento (14); en nuestro medio la vancomicina se revela como un antibiótico de gran actividad.Las resistencias entre los bacilos gram negativos están en aumento con publicación de cepas de Klebsiella sp con EBSL de origen en unidades de críticos y posterior difusión en el medio hospitalario $(15,16)$. La resistencia a quinolonas entre los gram negativos es un problema en aumento, más acusado en la comunidad (18\% en nuestra serie) debido a su extendido uso en medio extrahospitalario. Para la Pseudomonas aeruginosa y Acinetobacter se están detectando resistencia a carbapenemes, problema ausente en nuestro medio.

Destacar la necesidad de considerar como bacteriemia verdadera, a la luz de los resultados, dos microorganismos discutidos tradicionalmente. El aislamiento de Candida no debería ser considerado como una colonización transitoria por la elevada mortalidad que presenta y porque no existe modo de reconocer aquellas que desarrollaran la candidiasis diseminada; se impone el tratamiento al disponer actualmente de antifúngicos de baja toxicidad $(17,18)$. Por otro lado se ha discutido la importancia de SCN como patógeno nosocomial, pero ya estudios recientes han mostrado que la positividad de un hemocultivo se asocia con episodios clínicamente relevantes $(19,20)$.

En nuestra serie los aislamientos de $\mathrm{SCN}$, se asocian a una mortalidad más elevada de la esperada para la literatura, lo que resulta discordante con el carácter "relativamente benigno de la bacteriemia". Cuando analizamos las posibles causas de esta mortalidad elevada objetivamos que hasta un $49 \%$ de los episodios recibieron tratamiento empírico inadecuado, unas tasas de inadecuación muy superiores al resto de microorganismos (p.000), lo que nos obliga a modificar actitudes en cuanto a su consideración diagnóstica y tratamiento específico.

La inadecuación del tratamiento antibiótico definido es un factor importante en la emergencia de infecciones debidas a bacterias resistentes, que se podría minimizar estableciendo consultas con especialistas de infecciología y guías antibióticas adecuadas a los gérmenes más prevalentes (21).

En conclusión. En nuestro medio los gram positivos presentan elevadas tasas de resistencia con rangos de MARSA similares a hospitales de mayores dimensiones. Se mantiene la sensibilidad universal a la vancomicina. Los gramnegativos de predominio comunitario presentan bajas tasas de EBSL. Una llamada de atención sobre las Pseudomonas multiresistentes de las unidades de agudos con limitadas opciones terapéuticas. Estos datos deberían tenerse en cuenta en el momento de la elección del tratamiento empírico inicial, uno de los condicionantes de supervivencia.

\section{Bibliografía}

1. Weinstein MP, Towns ML, Quartey SM, Mirret S, Reimer LG, Parmigiani G, Reller B. The clinical significance of positive blood cultures in the 1990s: a prospective comprehensive evaluation of microbiology, epidemiology and outcome of bacteremia and fungemia in adults. Clin Infect Dis 24: 584-602.

2. Pfaller MA, Jones RN, Doren GV, Kugler K and The SENTRY Participants Group. Bacterial Pathogens Isolated from Patients with Bloodstream Infection: Frequencies of Occurrence and Antimicrobial Susceptibility Patterns from the SENTRY Antimicrobial Surveillance Program (United States and Canada, 1997). Antimicrob Agents Chemother July 1998; 42: 1762-70.

3. Archibald L, Phillips L, Monnet D, McGowan JE Jr, Tenover F, Gaynes R. Antimicrobial resistance in isolates from in-patients and outpatients in the United States: increasing importance of the intensive care unit. Clin Infect Dis 1997; 24: 211-5.

4. Ballow C.H and Schentag JJ Trends in antibiotic utilization and bacterial resistance: report of the National Nosocomial Resistance Surveillance. Group Diagn Microbiol Infect Dis 1992; 15: 37S-42S.

5. Tenover FC, Hughes JM. The challenges of emerging infectious diseases: development and spread of multiply-resistant bacterial pathogens. JAMA 1996, 275: 300-4.

6. Pazos R, Fernández R, Paz I, Tinajas A, Cantón I, Abel V, González R, Martínez R, Gayoso P. Factores pronósticos de bacteraemia: estudio prospective. An Med Interna (Madrid) 2001; 18: 41-6.

7. National Committee for Clinical Laboratory Standards (NCLSS). Performance standards for antimicrobial susceptibility testing. Supplement tables. NCCLS document M100-S8. Wayne, PA: NCCLS 1998 Mc Cabe WR, Jackson GG. Gram negative bacteremia. Etiology and ecology. Arch Intern Med 1962; 110: 847-55.

8. Vázquez F, Mendoza MC, Villar MH, Pérez F, Méndez FJ. Survey of bacteraemia in a Spanish Hospital over a decade (1981-1990). J Hosp Infect 1994; 26: 111-21.

9. Archibald L, Jones RN. The emergent needs for basic research, education and surveillance of antimicrobial resistance. Diagn Microbiol Infect Dis 1996; 25: 1-9.

10. Centres for Disease Control and Prevention. Nosocomial enterococci resistant to vancomycin- United states, 1989-1993- MMWR Morb Mortal Wkly Rep 1993; 42: 597-9.

11. Fluit AC, Jones ME, Schmitz FJ, Acar J, Gupta R, Verhoef J, and the

SENTRY participants Group. Antimicrobial Susceptibility and Frequency of Occurrence of Clinical Blood Isolates in Europe from the SENTRY Antimicrobial Surveillance Program, 1997 and 1998. Clin Infec Dis 2000, 456-60.

12. Johnson LB, Bhan A, Pawlak J, Manzor O, Saravolatz LD. Changing epidemiology of community-onset methicillin-resistant Staphylococcus aureus bacteremia. Infect Control Hosp Epidemiol 2003; 24 (6): 431-5.

13. Hofmann J, Cetron MS, Farley MM, Baugman WS, Facklam RR, Elliott JA, et al. The prevalence of drug ressitant Streptococcus pneumoniae in Atlanta. N Engl J Med 1995; 33: 481-6

14. Mutnick AH, Biedenbach DJ, Jones RN. Geographic variations and trends in antimicrobial resistance among Enterococcus faecalis and Enterococcus faecium in th SENTRY Antimicrobial Surveillance Program (1997-2000). Diagn Microbial Infect Dis 2003; 46 (1): 63-8.

15. Monnet D, Edwards J, Gaynes R, the National Nosocomial Infections Surveillance System. Extended spectrum beta-lactam-resistant nosocomial Klebsiella pneumoniae: epidemiologic evidence of interhospital transmission (abstract no 24. In: program of the 4th annual meeting of the Society for Healthcare Epidemiology of America (New Orleans. Thorofare, NJ: SLACK, 1994: 23).

16. MacKenzie FM, Forbes KJ, Dorai-John T, Amyes SGB, Gould IM. Emergence of acquired carbapenem-resistant Klebsiella pneumoniae (letter). Lancet 1997; 351: 783.

17. Edwards JE Jr, Filler SG. Current strategies for treating invasive candidiasis: emphasis on infections in non neutropenic patients. Clin Infect Dis 1992; 14 (Supl. 1): S 106-13

18. Pittet D, Garbino J. Fungal infections in the critically ill. Curr Opin Crit Care 1995; 1: 369-80

19. Winston DJ, Dudnick DV, Chapin M. Coagulase-negative staphycoccal bacteremia in patients receiving immunosuppressive therapy. Arch Intern Med 1983; 143: 32-6.

20. Domínguez de Villota E, Algora-Weber A, Millian I, Rubio JJ, Galdos P, Mosquera JM. Early evaluation of coagulase negative staphylococcus in blood samples of intensive care unit patients. A clinically uncertain judgement. Intensive Care Med 1987; 13: 390-4.

21. Kollef $\mathrm{MH}$. Inadequate antimicrobial treatment: an important determination of outcome for hospitalised patients. Clin Infect Dis 2000; 31 (Supl. 4): S 131-8. 